\title{
Perturbation of TWO ELEMENTARY WAVES WITH INTERACTION
}

\author{
Liu Hongxia and Lin Longwei
}

\begin{abstract}
In this paper, we study the perturbation of two interacting elementary waves for the system of isentropic gas dynamics equations. The entropy solutions for the perturbed problems are obtained by Glimm's scheme. Under the condition that the initial data will issue two elementary waves with interaction, we conclude that the corresponding perturbed solutions exist if the total variation of the perturbation of the initial data is sufficiently small.
\end{abstract}

\section{Introduction}

Consider the Cauchy problem for the system of isentropic gas dynamics equations in Lagrangian coordinates:

$$
\begin{gathered}
v_{t}-u_{x}=0 \\
u_{t}+p(v)_{x}=0 \\
(u, v)(x, 0)=\left(u_{0}(x), v_{0}(x)\right) \quad x \in R, t>0,
\end{gathered}
$$

where $p(v) \in C^{2}(0,+\infty), p^{\prime}(v)<0, p^{\prime \prime}(v)>0$.

We study some perturbation problems for $(\mathbf{E})$ and $(\mathbf{I})$. Suppose the initial data (I) are perturbed; the perturbed initial data are

$$
(\bar{u}, \bar{v})(x, 0)=\left(\bar{u}_{0}(x), \bar{v}_{0}(x)\right) \quad x \in R
$$

where $\left\|\left(\bar{u}_{0}(x)-u_{0}(x), \bar{v}_{0}(x)-v_{0}(x)\right)\right\|$ is sufficiently small; $\|\cdot\|$ is a norm in a certain sense. The function defined by $\left(\bar{u}_{0}(x)-u_{0}(x), \bar{v}_{0}(x)-v_{0}(x)\right)$ is called the perturbation of the initial data $\left(u_{0}(x), v_{0}(x)\right)$.

Definition 1. The entropy solutions $(\bar{u}(x, t), \bar{v}(x, t))$ of the perturbed problem (E) and $(\overline{\mathbf{I}})$ are called perturbed solutions of the corresponding unperturbed problem $(\mathbf{E})$ and $(\mathbf{I})$.

Definition 2. The entropy solution $(u(x, t), v(x, t))$ of the Cauchy problem $(\mathbf{E})$ and (I) is called stable if

$$
\|(\bar{u}(x, t)-u(x, t), \bar{v}(x, t)-v(x, t))\| \rightarrow 0 \text { as }\left\|\left(\bar{u}_{0}(x)-u_{0}(x), \bar{v}_{0}(x)-v_{0}(x)\right)\right\| \rightarrow 0 .
$$

Obviously, the existence of perturbed solutions is an important prior condition for the study of stability.

In the celebrated paper by Glimm [6], his famous result can be seen as the stability to constant solutions for $n \times n$ nonlinear hyperbolic conservation laws. In [13], Wang

Received September 12, 1995, revised July 3, 1996.

1991 Mathematics Subject Classification: 35L60, 35L65.

Key words and phrases: isentropic gas dynamics equations, perturbed problem, elementary waves with interaction. 
and $\mathrm{Li}$ obtained the perturbed solutions to Riemann problems for the isentropic gas dynamics equations $(\mathbf{E})$. Since there is no interaction between the elementary waves in [13], this core result can be thought of as the perturbation of a single shock. The stability of the single shock for $n \times n$ nonlinear hyperbolic conservation laws was obtained by Chern [4]. Furthermore, under the Chern stability condition [4], Asakura [1] obtained asymptotic stability of solutions with a single shock for $n \times n$ systems. In $[2,3]$, Asakura studied asymptotic stability of solutions with strong shocks which do not interact with each other for the isothermal gas dynamics equations. If the solutions of the Cauchy problems for $2 \times 2$ nonlinear hyperbolic conservation laws only consist of rarefaction waves, then the corresponding perturbation problems have been solved by Liu [9] and Lin and Xie [8]

In this paper, the core result is about the perturbation of two elementary waves for $(\mathbf{E})$ and $(\mathbf{I})$. In other words, we study the existence of entropy solutions for the perturbed problem $(\mathbf{E})$ and $(\overline{\mathbf{I}})$ by Glimm's scheme where the unperturbed initial data (I) will produce two elementary waves with interaction.

\section{Properties of shock curves and estimates on interactions}

The characteristics of the system (E) are $\lambda_{1}=-\sqrt{-p^{\prime}(y)}=-\lambda_{2}$. The corresponding Riemann invariants can be taken as $r=u+\int_{1}^{v} \sqrt{-p^{\prime}(y)} d y$ and $s=u-\int_{1}^{v} \sqrt{-p^{\prime}(y)} d y$. The mapping from $(u, v), v>0$ to $(r, s), s-r>-2 \int_{1}^{v} \sqrt{-p^{\prime}(y)} d y$ is one-to-one and onto, so we may use $(u, v)$ or $(r, s)$ as the variables at our convenience.

To carry out our analysis, we improve the well-known framework given in [6] and $[10]$.

We now briefly describe Glimm's difference scheme [6]. Choose mesh lengths $l, h$ so that $l / h$ is a constant which is greater than $\max \left\{\left|\lambda_{1}\right|,\left|\lambda_{2}\right|\right\}$. In each step $n h<$ $t<(n+1) h$ the approximate solution is obtained exactly and consists of elementary waves generated at points of discontinuity $x=m l$ where $n \geq 0, m$ are integers. At time $t=(n+1) h$, the value of the approximate solution $\left(u^{l}(x, t), v^{l}(x, t)\right),(m-1) l<$ $x<(m+1) l$, is set to be the value of the exact solution in the strip $n h<t<(n+1) h$ at $t=(n+1) h$ and $x=\left(m+e_{n+1}\right) l$. Here $\left\{e_{n}\right\}$ is a prechosen random sequence in $(-1,1)$. The upper half $(x, t)$-plane is covered by diamonds $\triangle_{m, n}$ with vertices $a_{m, n-1}, a_{m+1, n}, a_{m, n+1}, a_{m-1, n}$, where the mesh point $a_{i, j}=\left(i+e_{j}, j\right), i+j=$ even. We consider curves consisting of line segments of the form $L_{m . n . m+1 . n+1}$ joining $a_{m, n}$ to $a_{m+1, n+1}$ and $L_{m . n . m+1 . n-1}$ joining $a_{m, n}$ to $a_{m+1, n-1}$. If the mesh index $m$ increases monotonically on such a curve, we call it an $I$-curve. Let $O$ denote the unique $I$-curve passing though the mesh point at $t=0$ and $t=h$. In order to obtain a uniform bound on the approximate solutions $\left(u^{l}(x, t), v^{l}(x, t)\right)$ in $0 \leq t \leq T, x \in R$, and a uniform bound on the total variation of $\left(u^{l}(\cdot, t), v^{l}(\cdot, t)\right)$ on each line $t=$ const $>0$, we consider the interactions of shocks and centered rarefaction waves occurring in the diamond $\triangle_{m, n}$ bounded by the $I$-curve $J_{1}$ and its immediate successor $J_{2}$.

If $R_{1}$ (resp., $S_{1}$ ) and $R_{2}$ (resp., $S_{2}$ ) are the rarefaction (resp., shock) waves corresponding to the first and second characteristic families respectively, then there are six nontrivial two elementary wave interactions [10]:

(i) $R_{2}$ interacts with $R_{1}$;

(ii) $S_{1}$ interacts with $S_{1}$ (or $S_{2}$ interacts with $S_{2}$ );

(iii) $R_{2}$ interacts with $S_{1}$ (or $S_{2}$ interacts with $R_{1}$ ); 
(iv) $S_{2}$ interacts with $S_{1}$;

(v) $S_{2}$ interacts with $R_{2}$ (or $R_{1}$ interacts with $S_{1}$ );

(vi) $R_{2}$ interacts with $S_{2}$ (or $S_{1}$ interacts with $R_{1}$ ).

Let $\beta$ (resp., $\gamma$ ) be the shock wave of the first (resp., second) kind on $J$. Let $b$ (resp., $g$ ) be the rarefaction wave of the first (resp., second) kind on $J$. We define the major strength, denoted by $|\cdot|$, of the shock, the minor strength, denoted by $|\cdot|_{-}$, of the shock, and the strength of the rarefaction waves, denoted by $|\cdot|$, as follows: $|\beta|=r_{l}-r_{r},|\beta|_{-}=s_{l}-s_{r},|b|=r_{r}-r_{l},|\gamma|=s_{l}-s_{r},|\gamma|_{-}=r_{l}-r_{r}$, $|g|=s_{r}-s_{l}$, where $\left(r_{l}, s_{l}\right)$ and $\left(r_{r}, s_{r}\right)$ denote the left state and right state of the waves, respectively.

In what follows, following [10], we denote by $\gamma+\beta \rightarrow \beta^{\prime}+\gamma^{\prime}$ the interaction of an $S_{2}$ and an $S_{1}$, which produces an $S_{1}$ and an $S_{2}$; the other cases are written in a similar way.

Following [10], the 1-shock curve $S_{1}$ and 2-shock curve $S_{2}$ with initial point $\left(r_{0}, s_{0}\right)$ can be written, respectively, as

$$
\begin{array}{ll}
s_{0}-s=g_{1}\left(r_{0}-r,\left(r_{0}, s_{0}\right)\right) & r<r_{0}, \\
r_{0}-r=g_{2}\left(s_{0}-s,\left(r_{0}, s_{0}\right)\right) & s<s_{0} .
\end{array}
$$

It is easy to prove:

Lemma 2.1. For the 1-shock curve $S_{1}$ in the region $P_{1} \subset \subset P_{0}\left(P_{0}=\{(r, s) \mid v>0\}\right)$ :

(i) $g_{1}^{\prime}\left(a,\left(r_{0}, s_{0}\right)\right) \in[0,1)$.

(ii) For every $\varepsilon>0$, there is a $\delta>0$ only depending on $\varepsilon$ and $p(v)$ such that $g_{1}^{\prime}\left(a,\left(r_{0}, s_{0}\right)\right)<\varepsilon$, as $a=r_{0}-r<\delta$.

(iii) $\inf _{P_{1}}\left(1-g_{1}^{\prime}\left(a,\left(r_{0}, s_{0}\right)\right)\right)>\bar{C}>0$, where $\bar{C}$ is a constant only depending on the region $P_{1}$ and $p(v)$.

(iv) $g_{1}^{\prime \prime}\left(a,\left(r_{0}, s_{0}\right)\right) \geq 0$.

We have similar conclusions for the 2-shock curve $S_{2}$.

For convenience, we introduce a more refined lemma in [8].

Lemma 2.2. Suppose that all waves considered below are contained in the region $P_{1} \subset \subset P_{0}$, in the $(r, s)$-plane. Then the following estimates are valid for the corresponding interactions.

(i) $\gamma+\beta \rightarrow \beta^{\prime}+\gamma^{\prime}$ :

||$\beta^{\prime}|-| \beta|| \leq C|\beta||\gamma|^{3}, \quad|| \gamma^{\prime}|-| \gamma|| \leq C|\gamma||\beta|^{3}$.

(ii) (1) $\gamma+b \rightarrow b^{\prime}+\gamma^{\prime}$ :

||$b^{\prime}|-| b|| \leq C|b||\gamma|^{3}, \quad\left|\gamma^{\prime}\right|=|\gamma|$.

(2) $g+\beta \rightarrow \beta^{\prime}+g^{\prime}$ :

||$g^{\prime}|-| g|| \leq C|g||\beta|^{3}, \quad\left|\beta^{\prime}\right|=|\beta|$.

(iii) (1) $\gamma_{1}+\gamma_{2} \rightarrow b^{\prime}+\gamma^{\prime}$ :

$\left|b^{\prime}\right| \leq C\left|\gamma_{1}\right|\left|\gamma_{2}\right|\left(\left|\gamma_{1}\right|+\left|\gamma_{2}\right|\right), \quad\left|\gamma^{\prime}\right|=\left|\gamma_{1}\right|+\left|\gamma_{2}\right|$.

(2) $\beta_{1}+\beta_{2} \rightarrow \beta^{\prime}+g^{\prime}$ :

$\left|g^{\prime}\right| \leq C\left|\beta_{1}\right|\left|\beta_{2}\right|\left(\left|\beta_{1}\right|+\left|\beta_{2}\right|\right), \quad\left|\beta^{\prime}\right|=\left|\beta_{1}\right|+\left|\beta_{2}\right|$. 
(iv) (1) $g+\gamma \rightarrow \beta^{\prime}+\gamma^{\prime}$ :

$\left|\gamma^{\prime}\right|-|\gamma| \leq-\left|\beta^{\prime}\right|\left(1+C|\gamma|^{-2}\right), \quad\left|\beta^{\prime}\right| \leq C|\gamma|^{3}$.

(2) $g+\gamma \rightarrow \beta^{\prime}+g^{\prime}:$

$\left|g^{\prime}\right|-|g| \leq|\gamma|\left(C|\gamma|^{8}-1\right), \quad\left|\beta^{\prime}\right| \leq C|\gamma|^{3}$.

(3) $\beta+b \rightarrow \beta^{\prime}+\gamma^{\prime}$ :

$\left|\beta^{\prime}\right|-|\beta| \leq-\left|\gamma^{\prime}\right|\left(1+C|\beta|^{-2}\right), \quad\left|\gamma^{\prime}\right| \leq C|\beta|^{3}$.

(4) $\beta+b \rightarrow b^{\prime}+\gamma^{\prime}$ :

$\left|b^{\prime}\right|-|b| \leq|\beta|\left(C|\beta|^{8}-1\right), \quad\left|\gamma^{\prime}\right| \leq C|\beta|^{3}$.

(v) (1) $\gamma+g \rightarrow \beta^{\prime}+\gamma^{\prime}$ :

There exist $\beta_{0}, \gamma_{0}$ such that the interaction

$\gamma_{0}+\beta_{0} \rightarrow \beta^{\prime}+\gamma^{\prime}$ is the same as in (i) and

$\left|\beta_{0}\right|+\left|\gamma_{0}\right|-|\gamma| \leq-C\left|\beta_{0}\right||\gamma|^{-2}, \quad\left|\beta_{0}\right| \leq C|\gamma|^{3}$.

(2) $\gamma+g \rightarrow \beta^{\prime}+g^{\prime}$ :

$\left|g^{\prime}\right|-|g| \leq|\gamma|\left(C|\gamma|^{8}-1\right), \quad\left|\beta^{\prime}\right| \leq C|\gamma|^{3}$.

(3) $b+\beta \rightarrow \beta^{\prime}+\gamma^{\prime}$ :

$\left|\beta_{0}\right|-|\beta| \leq-\left|\gamma_{0}\right|\left(1+C|\beta|^{-2}\right), \quad\left|\gamma_{0}\right| \leq C|\beta|^{3}$

where $\beta_{0}, \gamma_{0}$ are defined as in (1).

(4) $b+\beta \rightarrow b^{\prime}+\gamma^{\prime}$ :

$\left|b^{\prime}\right|-|b| \leq|\beta|\left(C|\beta|^{8}-1\right), \quad\left|\gamma^{\prime}\right| \leq C|\beta|^{3}$.

(vi) $g+b \rightarrow b^{\prime}+g^{\prime}$ :

$\left|g^{\prime}\right|=|g|, \quad\left|b^{\prime}\right|=|b|$.

Here $C$ is a generic constant which only depends on the region $P_{1}$ and $p(v)$.

If both waves with interaction are not small, we have the following lemma of estimates on the interaction:

Lemma 2.3. $[6,8]$ Under the same assumption as in Lemma 2.2, the following estimates are valid for the corresponding interactions.

(1) $\gamma+\beta \rightarrow \beta^{\prime}+\gamma^{\prime}: \quad\left|\gamma^{\prime}\right| \leq\left(1+C_{1}|\beta|\right)|\gamma|$.

(2) $\beta_{1}+\beta_{2} \rightarrow \beta^{\prime}+g^{\prime}: \quad\left|g^{\prime}\right| \leq C_{1}\left|\beta_{1}\right|\left|\beta_{2}\right|$.

(3) $b+\beta \rightarrow \beta^{\prime}+\gamma^{\prime}: \quad\left|\gamma^{\prime}\right| \leq C_{1}|\beta||b|$.

(4) $g+\beta \rightarrow \beta^{\prime}+g^{\prime}: \quad\left|g^{\prime}\right| \leq\left(1+C_{1}|\beta|\right)|g|$.

(5) $\beta+b \rightarrow \beta^{\prime}+\gamma^{\prime}: \quad\left|\gamma^{\prime}\right|+\left|\beta^{\prime}\right|-|\beta| \leq-C_{0}\left|\gamma^{\prime}\right|$.

(6) $g+b \rightarrow b^{\prime}+g^{\prime}: \quad\left|g^{\prime}\right|=|g|,\left|b^{\prime}\right|=|b|$.

Here $C_{0}, C_{1}$ are positive constants only depending on $p(v)$ and the region $P_{1}$.

We can obtain similar estimates for the remaining cases.

Remark 2.1. In the bounded region, the key point about Glimm's interaction estimates is whether they are suitable to small waves. Once the estimates are true for small waves, they also are true for large waves. Since the region considered here is bounded, Lemma 2.3 is obviously true. 
In view of (i) and (iii) in Lemma 2.1 and (5) in Lemma 2.3, it follows that there exists a constant $D>1$ such that

$$
\begin{gathered}
\sup _{P_{1}} g_{i}^{\prime}\left(a,\left(r_{0}, s_{0}\right)\right)<\frac{D-1}{D} \quad(i=1,2) \\
\frac{1}{1+C_{0}}<\frac{D-1}{D} .
\end{gathered}
$$

For interactions between a large wave and a small wave belonging to the same characteristic family, we have

Lemma 2.4. Suppose that all waves considered below are contained in the region $P_{1}$. Then the following estimates are valid for the corresponding interactions:

(i) $\beta+\beta_{1} \rightarrow \beta^{\prime}+g^{\prime}: \quad\left|g^{\prime}\right|<\frac{D-1}{D}\left|\beta_{1}\right|$.

(ii) $\beta+b \rightarrow \beta^{\prime}+\gamma^{\prime}:$ If $g_{2}^{\prime}\left(\frac{|b|}{C_{0}},\left(r_{0}, s_{0}\right)\right)<C_{0}-\frac{1}{D-1}$, for $\left(r_{0}, s_{0}\right) \in P_{1}$, then $\left|\gamma^{\prime}\right|<\frac{D-1}{D}|b|$.

There is no difficulty in proving this lemma. We can get similar conclusions for interactions between two waves belonging to the second characteristic family.

\section{Existence of perturbed solutions}

In this section, we shall study perturbation problems of two elementary waves with interaction for (E). Namely, we shall prove existence theorems for the perturbed solutions of $(\mathbf{E})$ with $(\mathbf{I})$, where the initial data $(\mathbf{I})$ generate two elementary waves with interaction.

In the $(r, s)$-plane, the initial data corresponding to $(\mathbf{I})$ are

$$
(r, s)(x, 0)=\left(r_{0}(x), s_{0}(x)\right) \quad x \in R
$$

where $\left(r_{0}(x), s_{0}(x)\right)=\left(r\left(u_{0}(x), v_{0}(x)\right), s\left(u_{0}(x), v_{0}(x)\right)\right)$. The perturbed initial data of (3.1) are

$$
(\bar{r}, \bar{s})(x, 0)=\left(r_{0}(x)+r_{1}(x), s_{0}(x)+s_{1}(x)\right)
$$

where $\left(r_{1}(x), s_{1}(x)\right)$ is the perturbation of the initial data $\left(r_{0}(x), s_{0}(x)\right)$ in the $(r, s)$ plane. We assume $\left(r_{1}(x), s_{1}(x)\right)$ satisfies

$$
\begin{gathered}
\operatorname{TV}\left\{r_{1}(x), s_{1}(x)\right\}<\varepsilon, \\
\lim _{x \rightarrow \pm \infty}\left(r_{1}(x), s_{1}(x)\right)=(0,0),
\end{gathered}
$$

where TV denotes the total variation and $\varepsilon>0$ is a sufficiently small constant only dependent on $p(v),\left(r_{0}(x), s_{0}(x)\right)$, and the region $P_{1}$.

First, we study the case in which the unperturbed initial data (I) will generate two shock waves belonging to the same characteristic family. For definiteness, we assume that the two shock waves are 1-shock waves.

Theorem 3.1. Suppose that the unperturbed initial data $\left(u_{0}(x), v_{0}(x)\right)$ consist of three pieces of constant functions generating two given 1-shock waves $x_{1}(t)$ and $x_{2}(t)$, where $x_{2}(t)$ lies to the right of $x_{1}(t)$ and the strengths of $x_{1}(t)$ and $x_{2}(t)$ are $\left|r_{1}^{0}\right|=r_{-}-r_{m}$ and $\left|r_{2}^{0}\right|=r_{m}-r_{+}$, respectively (here the state $(-)$is the left state of $x_{1}(t),(+)$ is the right state of $x_{2}(t)$, and $(m)$ is the state between $x_{1}(t)$ and $\left.x_{2}(t)\right)$. We assume the small perturbation has this form of initial data. If the perturbation $\left(r_{1}(x), s_{1}(x)\right)$ 
of the initial data $\left(r_{0}(x), s_{0}(x)\right)$ in the $(r, s)$-plane satisfies (3.3) and (3.4), then there exists a perturbed solution of the corresponding unperturbed problem (E) with (I).

Remark 3.1. For this unperturbed problem $(\mathbf{E})$ with $(\mathbf{I})$ and the unperturbed problem which will be discussed in the next theorem, the authors in papers [7, 11, 14] proved the existence of unperturbed solutions and gave their structure and properties.

From (3.3), we know that $\operatorname{TV}\left\{r_{1}(x), s_{1}(x)\right\}$ is sufficiently small. This means that comparing the strengths of $x_{1}(t)$ and $x_{2}(t), \operatorname{TV}\left\{r_{1}(x), s_{1}(x)\right\}$ is small. For definiteness, we refer to $x_{1}(t)$ and $x_{2}(t)$ as "large" shock waves. Throughout this paper, the "large" shock waves have similar meanings. From now on, we stipulate that the "small" perturbation is the perturbation of the initial data satisfying (3.3) and (3.4).

Proof of Theorem 3.1. According to Glimm's scheme [6], we only need to prove that the decreasing variation of the approximate perturbed solutions $\left(\bar{u}^{l}(x, t), \bar{v}^{l}(x, t)\right)$ is uniformly bounded for any $t>0$. We denote by $x_{1}^{l}(t)$ and $x_{2}^{l}(t)$ the perturbed approximate large 1-shock waves of $x_{1}(t)$ and $x_{2}(t)$, respectively (Figure 1 ).

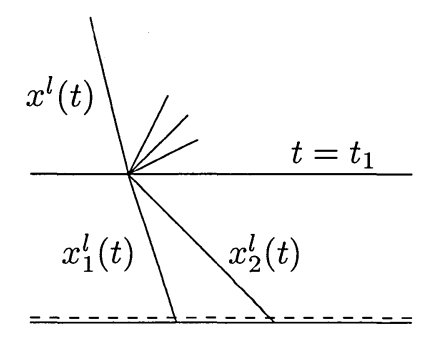

FIGURE 1

Let $t=t_{1}$ denote the time at which $x_{1}^{l}(t)$ interacts with $x_{2}^{l}(t), x^{l}(t)$ denote the approximate large 1-shock wave produced by the interaction of $x_{1}^{l}(t)$ and $x_{2}^{l}(t)$, and $J_{\left(t_{1}-h\right)}$ (or $\left.J_{\left(t_{1}+h\right)}\right)$, where $h$ is the time mesh, denote the $I$-curve between $t=t_{1}$ and $t=t_{1}-h$ (or $t=t_{1}+h$ ). Now we design uniformly small functionals on any $I$-curve $J$ on $0 \leq t \leq t_{1}$ and $t \geq t_{1}$, respectively, to dominate the total sum of the strengths of all small shock waves crossing $J$ (here those shock waves except $x_{i}^{l}(t)(i=1,2)$ and $x^{l}(t)$ are defined as small shock waves). In the following definitions of the functionals, the strengths of the 2-rarefaction waves are not considered because the interaction of $x_{1}^{l}(t)$ and $x_{2}^{l}(t)$ will generate a large 2-rarefaction wave, whereas functionals with the strengths of 2-rarefaction waves cannot be uniformly small.

(I) When an $I$-curve $J$ lies on the plane $0 \leq t \leq t_{1}$, we define the functionals on $J$ :

$$
\begin{aligned}
F_{1}^{(s)}(J) & =\sum_{i=1}^{3} F_{0}\left(J^{i}\right) \\
F_{0}\left(J^{i}\right) & =M_{i} L_{0}\left(J^{i}\right)+Q_{0}\left(J^{i}\right) \quad(i=1,2,3)
\end{aligned}
$$


where $J^{1}$ (resp., $J^{3}$ ) is the part of $J$ lying to the left (resp., right) of $x_{1}^{l}(t)$ (resp., $\left.x_{2}^{l}(t)\right), J^{2}$ is the part of $J$ between $x_{1}^{l}(t)$ and $x_{2}^{l}(t)$, and

$$
\begin{aligned}
& L_{0}\left(J^{i}\right)=\sum\left\{|\alpha|: \alpha \in J^{i}, \alpha \text { is a shock wave or 1-rarefaction wave }\right\}(i=1,2,3), \\
& Q_{0}\left(J^{i}\right)=\sum\left\{\left|\alpha_{j}\right|\left|\alpha_{k}\right|: \alpha_{j} \in J^{i}, \alpha_{k} \in \bigcup_{i=1}^{3} J^{i}, \quad \alpha_{j}, \alpha_{k}\right. \text { are not }
\end{aligned}
$$

2-rarefaction waves and approach each other $\}(i=1,2,3)$,

$$
M_{i}=\prod_{j=i}^{2}\left(1+4 C_{1}\left|r_{j}^{0}\right|\right) \quad(i=1,2), \quad M_{3}=1
$$

where $C_{1}$ is a constant appearing in Lemma 2.3 and $\alpha \in J^{i}$ means that $\alpha$ is a wave crossing $J^{i}(i=1,2,3)$. If there is no wave crossing $J^{i}$, then we stipulate $F_{0}\left(J^{i}\right)=0$ $(i=1,2,3)$.

(II) When any $I$-curve $J$ is on $t \geq t_{1}$, we define

$$
F_{2}^{(s)}(J)=F_{0}\left(J^{1}\right)+F_{0}\left(J^{3}\right)
$$

where $J^{1}$ (resp., $J^{3}$ ) is the part of $J$ lying to the left (resp., right) of $x^{l}(t)$. The definition of $F_{0}\left(J^{i}\right)(i=1,3)$ is consistent with that of (I).

If $x_{1}^{l}(t)$ does not interact with $x_{2}^{l}(t)$ forever, then there is no need to define $F_{2}^{(s)}(J)$.

We shall prove by induction that

(A) When $J$ lies on $0 \leq t \leq t_{1}, F_{1}^{(s)}(J) \leq F_{1}^{(s)}(O)<\eta$ and the total sum of the strengths of all shock waves crossing $J$ is $L_{s}(J)<\left|r^{0}\right|+12 \varepsilon e^{4 C_{1}\left|r^{0}\right|}$.

(B) When $J$ lies on $t \geq t_{1}, F_{2}^{(s)}(J) \leq F_{1}^{(s)}(O)<\eta$ and the total sum of the strengths of all shock waves crossing $J$ is $L_{s}(J)<\left|r^{0}\right|+12 \varepsilon e^{4 C_{1}\left|r^{0}\right|}$.

Here $\eta=4 \varepsilon e^{4 C_{1}\left|r^{0}\right|},\left|r^{0}\right|=\left|r_{1}^{0}\right|+\left|r_{2}^{0}\right|$, and $\varepsilon$ is a sufficiently small constant appearing in (3.3).

The proof will be separated into two steps.

Step I Consider the problem on $0 \leq t \leq t_{1}$.

$$
\begin{aligned}
F_{1}^{(s)}(O) & =\sum_{i=1}^{3} M_{i} L_{0}\left(O^{i}\right)+\sum_{i=1}^{3} Q_{0}\left(O^{i}\right) \\
& \leq\left(M_{1}+\sum_{i=1}^{3} L_{0}\left(O^{i}\right)\right) \sum_{i=1}^{3} L_{0}\left(O^{i}\right) \\
& <2 \varepsilon\left(M_{1}+2 \varepsilon\right)<4 \varepsilon e^{4 C_{1}\left|r^{0}\right|}=\eta
\end{aligned}
$$

when $\varepsilon<1 / 2$.

Let $\left(r_{-}^{l}(t), s_{-}^{l}(t)\right)$ and $\left(r_{m}^{l}(t), s_{m}^{l}(t)\right)$ denote the left and right states of $x_{1}^{l}(t)$ in the strip $0<t<h$, respectively, then

$$
\left|r_{-}^{l}(t)-r_{-}\right| \leq F_{0}\left(O^{1}\right), \quad\left|r_{m}^{l}(t)-r_{m}\right| \leq F_{0}\left(O^{2}\right) .
$$

Therefore, when $\eta<\left|r_{1}^{0}\right|$, in the strip $0<t<h, x_{1}^{l}(t)$ is a 1-shock wave and

$$
\begin{aligned}
0<\left|r_{1}^{0}\right|-\eta & <\left|r_{1}^{0}\right|-\left(F_{0}\left(O^{1}\right)+F_{0}\left(O^{2}\right)\right) \leq\left|x_{1}^{l}(t)\right| \\
& \leq\left|r_{1}^{0}\right|+F_{0}\left(O^{1}\right)+F_{0}\left(O^{2}\right)<\left|r_{1}^{0}\right|+\eta<2\left|r_{1}^{0}\right| .
\end{aligned}
$$


Similarly, we can prove that when $\eta<\left|r_{2}^{0}\right|$, in the strip $0<t<h, x_{2}^{l}(t)$ is a 1-shock wave and

$$
\begin{aligned}
0<\left|r_{2}^{0}\right|-\eta & <\left|r_{2}^{0}\right|-\left(F_{0}\left(O^{2}\right)+F_{0}\left(O^{3}\right)\right) \leq\left|x_{2}^{l}(t)\right| \\
& \leq\left|r_{2}^{0}\right|+F_{0}\left(O^{2}\right)+F_{0}\left(O^{3}\right)<\left|r_{2}^{0}\right|+\eta<2\left|r_{2}^{0}\right| .
\end{aligned}
$$

Suppose that any $I$-curve $J_{1}$ and its immediate successor $J_{2}$ lie on $0 \leq t \leq t_{1}$, $F_{1}^{(s)}\left(J_{1}\right) \leq F_{1}^{(s)}(O)<\eta, x_{1}^{l}(t)$ and $x_{2}^{l}(t)$ crossing $J_{1}$ are 1-shock waves, and the strength of $x_{i}^{l}(t)(i=1,2)$ satisfies the following inequality:

$$
\begin{aligned}
0<\left|r_{i}^{0}\right|-\eta & <\left|r_{i}^{0}\right|-\left(F_{0}\left(J_{1}^{i}\right)+F_{0}\left(J_{1}^{i+1}\right)\right) \leq\left|x_{i}^{l}(t)\right| \\
& \leq\left|r_{i}^{0}\right|+F_{0}\left(J_{1}^{i}\right)+F_{0}\left(J_{1}^{i+1}\right)<\left|r_{i}^{0}\right|+\eta<2\left|r_{i}^{0}\right| .
\end{aligned}
$$

We shall prove $F_{1}^{(s)}\left(J_{2}\right) \leq F_{1}^{(s)}\left(J_{1}\right), x_{i}^{l}(t)(i=1,2)$ crossing $J_{2}$ is a 1-shock wave, and (3.7) is valid for $J_{2}$, i.e., if $J_{2}$ takes the place of $J_{1}$ in (3.7), then the inequality also is valid. For this, we consider all possible types of interactions of waves in the diamond $\Delta$ bounded by $J_{1}$ and $J_{2}$.

It is well-known that the interaction of three waves can be reduced to a sequence of interactions of two waves for the system of isentropic gas dynamics equations. For example, Wang and $\mathrm{Li}$ [13] discussed this problem for the polytropic gas $p=$ $k^{2} v^{-\gamma}$ (here $k>0$ and $\gamma \geq 1$ are constants). Their discussion is applicable to general $p$ systems and can be generalized with no revision. Therefore, without loss of generality, we may study the interaction of two waves coming into the diamond $\Delta$.

(i) $x_{1}^{l}(t)$ and $x_{2}^{l}(t)$ are not in $\Delta$. Then the interactions in $\Delta$ are among the small waves. Using Lemma 2.2 , we obtain $F_{1}^{(s)}\left(J_{2}\right) \leq F_{1}^{(s)}\left(J_{1}\right)$ provided

$$
0<\eta<\min \left\{1, \sqrt[3]{C},\left(4 C e^{4 C_{1}\left|r^{0}\right|}\right)^{-1}\right\}
$$

where $C$ is a constant appearing in Lemma 2.2.

(ii) $x_{1}^{l}(t)$ is in $\Delta$, but $x_{2}^{l}(t)$ is not in $\Delta$.

$$
\begin{aligned}
(1) \beta+\beta_{1} \rightarrow \beta^{\prime}+g^{\prime} & \left(\beta=x_{1}^{l}(t), \beta_{1} \neq x_{2}^{l}(t), \text { Figure } 2\right) . \\
& F_{0}\left(J_{2}^{1}\right)=F_{0}\left(J_{1}^{1}\right) \\
& F_{0}\left(J_{2}^{3}\right)=F_{0}\left(J_{1}^{3}\right) \\
& F_{0}\left(J_{2}^{2}\right)-F_{0}\left(J_{1}^{2}\right) \leq-M_{2}\left|\beta_{1}\right| .
\end{aligned}
$$

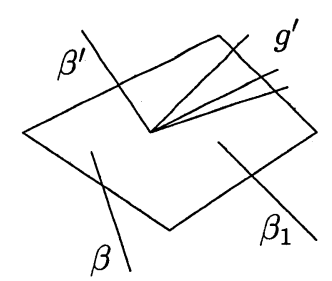

Figure 2

Thus $F_{1}^{(s)}\left(J_{2}\right)-F_{1}^{(s)}\left(J_{1}\right) \leq 0$. 
(2) $\beta+b \rightarrow \beta^{\prime}+\gamma^{\prime}\left(\beta=x_{1}^{l}(t)\right.$, Figure 3$)$.

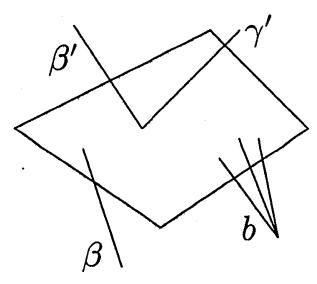

FiguRE 3

By (iv) in Lemma 2.1, we get

$$
g_{2}^{\prime}\left(\frac{|b|}{C_{0}},\left(r_{0}, s_{0}\right)\right) \leq g_{2}^{\prime}\left(\frac{\eta}{C_{0}},\left(r_{0}, s_{0}\right)\right)
$$

where $\left(r_{0}, s_{0}\right) \in P_{1}$ and $C_{0}$ is a constant appearing in Lemma 2.3. From (ii) in Lemma 2.1 , there is a constant $d>0$ only depending on $p(v)$ and $C_{0}-\frac{1}{D-1}$ such that

$$
g_{2}^{\prime}\left(\frac{|b|}{C_{0}},\left(r_{0}, s_{0}\right)\right)<C_{0}-\frac{1}{D-1} \text { when } \varepsilon<\frac{d C_{0}}{4} e^{-4 C_{1}\left|r^{0}\right|}
$$

In view of (ii) in Lemma 2.4, we obtain

$$
\left|\gamma^{\prime}\right|<\frac{D-1}{D}|b| .
$$

Since $F_{0}\left(J_{2}^{1}\right)=F_{0}\left(J_{1}^{1}\right)$ and $F_{0}\left(J_{2}^{3}\right)=F_{0}\left(J_{1}^{3}\right)$,

$$
\begin{aligned}
F_{0}\left(J_{2}^{2}\right)-F_{0}\left(J_{1}^{2}\right) & =M_{2}\left(\left|\gamma^{\prime}\right|-|b|\right)+\left|\gamma^{\prime}\right| \sum\left|\alpha_{k}\right|-|b| \sum\left|\alpha_{j}\right| \\
& \leq \frac{|b|}{D}\left((D-1) \eta-M_{2}\right) \leq 0
\end{aligned}
$$

when $(D-1) \eta<1$. Then $F_{1}^{(s)}\left(J_{2}\right)-F_{1}^{(s)}\left(J_{1}\right) \leq 0$.

(3) $\gamma+\beta \rightarrow \beta^{\prime}+\gamma^{\prime}\left(\beta=x_{1}^{l}(t)\right)$.

By (1) in Lemma 2.3, $\left|\gamma^{\prime}\right| \leq\left(1+C_{1}|\beta|\right)|\gamma|$,

$$
\begin{aligned}
F_{1}^{(s)}\left(J_{2}\right)-F_{1}^{(s)}\left(J_{1}\right) & =M_{2}\left|\gamma^{\prime}\right|+\left(\left|\gamma^{\prime}\right|-|\gamma|\right) \sum\left|\alpha_{k}\right|-M_{1}|\gamma| \\
& \leq M_{2}\left(1+C_{1}|\beta|\right)|\gamma|+C_{1}|\beta||\gamma| F_{1}^{(s)}(O)-M_{1}|\gamma| \\
& \leq\left(M_{2}\left(1+2 C_{1}|\beta|\right)-M_{1}\right)|\gamma| \quad \text { when } \eta<1 .
\end{aligned}
$$

By (3.7), $|\beta|<2\left|r_{1}^{0}\right|$; then

$$
F_{1}^{(s)}\left(J_{2}\right)-F_{1}^{(s)}\left(J_{1}\right) \leq\left(M_{2}\left(1+4 C_{1}\left|r_{1}^{0}\right|\right)-M_{1}\right)|\gamma|=0 .
$$

(4) $g+\beta \rightarrow \beta^{\prime}+g^{\prime}\left(\beta=x_{1}^{l}(t)\right)$.

(5) $b+\beta \rightarrow \beta^{\prime}+\gamma^{\prime}\left(\beta=x_{1}^{l}(t)\right)$.

(6) $\beta_{1}+\beta \rightarrow \beta^{\prime}+g^{\prime}\left(\beta=x_{1}^{l}(t)\right)$.

The proofs of (4)-(6) are similar to that of (3). 
In view of $(1)-(6)$, we have $F_{1}^{(s)}\left(J_{2}\right) \leq F_{1}^{(s)}\left(J_{1}\right)$.

Similar to the proof of (3.5), we can prove (3.7) is valid for $J_{2}$, and $x_{1}^{l}(t)$ crossing $J_{2}$ is a 1 -shock wave.

(iii) $x_{2}^{l}(t)$ is in $\Delta$, but $x_{1}^{l}(t)$ is not in $\Delta$. The proof is similar to that of (ii).

From (i)-(iii), we have $F_{1}^{(s)}(J) \leq F_{1}^{(s)}(O)<\eta$ for any $I$-curve $J$ on $0 \leq t \leq t_{1}$, and (3.7) is valid for $J$ (in particular, we have $\left.F_{1}^{(s)}\left(J_{\left(t_{1}-h\right)}\right)<\eta\right)$. Thus by (3.7), the total sum of the strengths of all shock waves crossing $J$ is

$$
\begin{aligned}
L_{s}(J) & \leq\left|x_{1}^{l}(t)\right|+\left|x_{2}^{l}(t)\right|+F_{1}^{(s)}(J) \\
& \leq\left|r^{0}\right|+3 F_{1}^{(s)}(J)<\left|r^{0}\right|+12 \varepsilon e^{4 C_{1}\left|r^{0}\right|} .
\end{aligned}
$$

Step II Consider the problem on $t \geq t_{1}$.

First, we can show by using the techniques similar to the ones in Step I:

$$
\begin{aligned}
F_{2}^{(s)}\left(J_{\left(t_{1}+h\right)}\right) & =F_{0}\left(J_{\left(t_{1}+h\right)}^{1}\right)+F_{0}\left(J_{\left(t_{1}+h\right)}^{3}\right) \\
& \leq F_{0}\left(J_{\left(t_{1}-h\right)}^{1}\right)+F_{0}\left(J_{\left(t_{1}-h\right)}^{3}\right) \\
& =F_{1}^{(s)}\left(J_{\left(t_{1}-h\right)}\right)<\eta .
\end{aligned}
$$

Let $t=t_{1}$ be the initial line and $J_{\left(t_{1}+h\right)}$ be the initial $I$-curve. Similar to Step I, using (3.8), we can obtain $F_{2}^{(s)}(J) \leq F_{2}^{(s)}\left(J_{\left(t_{1}+h\right)}\right)<\eta$ for any $I$-curve $J$ on $t \geq t_{1}$; moreover, $x^{l}(t)$ crossing $J$ is a 1 -shock wave and

$$
\begin{aligned}
0 & <\left|r^{0}\right|-\eta<\left|r^{0}\right|-\left(F_{0}\left(J^{1}\right)+F_{0}\left(J^{3}\right)\right) \leq\left|x^{l}(t)\right| \\
& \leq\left|r^{0}\right|+F_{0}\left(J^{1}\right)+F_{0}\left(J^{3}\right)<\left|r^{0}\right|+\eta<2\left|r^{0}\right| .
\end{aligned}
$$

Thus, it follows from (3.9) that the total sum of the strengths of all shock waves crossing $J$ is

$$
\begin{aligned}
L_{s}(J) & \leq\left|x^{l}(t)\right|+F_{2}^{(s)}(J) \leq\left|r^{0}\right|+2 F_{2}^{(s)}(J) \\
& <\left|r^{0}\right|+12 \varepsilon e^{4 C_{1}\left|r^{0}\right|} .
\end{aligned}
$$

In view of Step I and Step II, according to $[6,12]$, the decreasing variation of the approximate perturbed solutions $\left(\bar{u}^{l}(\cdot, t), \bar{v}^{l}(\cdot, t)\right)$ is bounded uniformly for any $t \geq 0$ when

$$
0<\varepsilon<\min \left\{\frac{\left|r_{1}^{0}\right|}{B}, \frac{\left|r_{2}^{0}\right|}{B}, \frac{1}{B}, \frac{1}{C B^{2}}, \frac{\sqrt[3]{C}}{B}, \frac{d C_{0}}{B}, \frac{1}{(D-1) B}\right\}
$$

where $B=4 e^{4 C_{1}\left|r^{0}\right|}$.

For the case in which the unperturbed data $(\mathbf{I})$ generates a 2-centered rarefaction wave (or 2-shock wave) and a 1-shock wave (or 1-centered rarefaction wave) which will interact, we state the existence theorem for the perturbed solution and give the definition of a uniformly small functional. The proof of the theorem is similar to that for Theorem 3.1.

Theorem 3.2. Suppose that the unperturbed initial data $\left(u_{0}(x), v_{0}(x)\right)$ consists of three pieces of constant functions generating a 2-centered rarefaction wave (or 2-shock wave) and a 1-shock wave $x(t)$ (or 1-centered rarefaction wave) which will interact. If we give a small perturbation to $\left(u_{0}(x), v_{0}(x)\right)$, then there exists a perturbed solution of the corresponding unperturbed problem (E) and (I). 
Let $x^{l}(t)$ denote the perturbed approximate large 1-shock wave of $x(t)$. We design a uniformly small functional $F_{1}^{(s)}(J)$ on any $I$-curve $J$ on the upper half-plane to control the total sum of the strengths of all small shock waves crossing $J$. Let

$$
\begin{aligned}
F_{1}^{(s)}(J) & =F_{0}\left(J^{1}\right)+F_{0}\left(J^{2}\right), \\
F_{0}\left(J^{i}\right) & =M_{i} L_{0}\left(J^{i}\right)+Q_{0}\left(J^{i}\right) \quad i=1,2, \\
M_{1} & =1+4 C_{1}\left|r^{0}\right|, \quad M_{2}=0
\end{aligned}
$$

where $J^{1}$ (resp., $J^{2}$ ) is the part of $J$ lying to the left (resp., right) of $x^{l}(t)$ and $\left|r^{0}\right|$ is the strength of $x^{l}(t) . L_{0}\left(J^{i}\right)$ and $Q_{0}\left(J^{i}\right), i=1,2$, are defined as in Theorem 3.1.

Now we turn to the case in which the unperturbed data (I) generates a 2 -shock wave and a 1-shock wave which will interact.

Theorem 3.3. Suppose that the unperturbed initial data $\left(u_{0}(x), v_{0}(x)\right)$ consist of three pieces of constant functions generating a 2-shock wave $x_{2}(t)$ and a 1-shock wave $x_{1}(t)$ where $x_{2}(t)$ lies to the left of $x_{1}(t)$. If we give a small perturbation to $\left(u_{0}(x)\right.$, $\left.v_{0}(x)\right)$, then there exists a perturbed solution of the corresponding unperturbed problem (E) and (I)

Remark 3.2. For this unperturbed problem and the unperturbed problem which will be discussed in the next theorem, Ding et al. [5] gave the existence, structure, and properties of the unperturbed solutions.

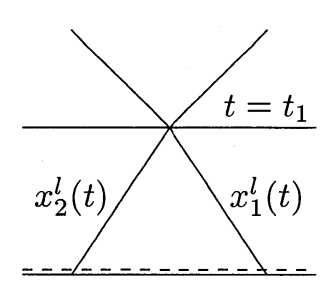

FIGURE 4

Proof of Theorem 3.3. Let $x_{2}^{l}(t)$ (resp., $x_{1}^{l}(t)$ ) denote the perturbed approximate large shock wave of $x_{2}(t)$ (resp., $x_{1}(t)$ ) (Figure 4). We assume $x_{2}^{l}(t)$ interacts with $x_{1}^{l}(t)$ at time $t=t_{1}$. We still denote by $x_{2}^{l}(t)$ (resp., $\left.x_{1}^{l}(t)\right)$ the penetrated approximate large 2 -shock wave (resp., 1-shock wave) (but $x_{1}^{l}(t)$ lies to the left of $x_{2}^{l}(t)$ ).

Let $J_{\left(t_{1}+h\right)}$ denote the $I$-curve between $t=t_{1}$ and $t=t_{1}+h$ ( $h$ is time mesh). For any $I$-curve $J$ on $0 \leq t \leq t_{1}$ and $t \geq t_{1}$, respectively, we design uniformly small functionals to control the total sum of the strengths of all small waves (i.e., all waves except $\left.x_{i}^{l}(t)(i=1,2)\right)$ crossing $J$. 
(I) When $J$ is on $0 \leq t \leq t_{1}+h$, we define on $J$ :

$$
\begin{aligned}
& F_{1}^{(s)}(J)=\sum_{i=1}^{3} F\left(J^{i}\right), \\
& F\left(J^{i}\right)=L\left(J^{i}\right)+Q\left(J^{i}\right) \quad i=1,3, \\
& F\left(J^{2}\right)=M L\left(J^{2}\right)+Q\left(J^{2}\right),
\end{aligned}
$$

where $J^{1}$ (or $J^{3}$ ) denotes the part of $J$ lying to the left (or right) of $x_{2}^{l}(t)$ (or $x_{1}^{l}(t)$ ) and $J^{2}$ the part of $J$ between $x_{2}^{l}(t)$ and $x_{1}^{l}(t)$. Moreover,

$$
\begin{aligned}
L\left(J^{i}\right) & =\sum\left\{|\alpha|: \alpha \in J^{i}, \text { and } \alpha \neq x_{1}^{l}(t), x_{2}^{l}(t)\right\} \quad i=1,3, \\
L\left(J^{1}\right) & =\sum\left\{|\alpha|: \alpha \in J^{1}\right\}, \\
Q\left(J^{i}\right) & =\sum\left\{\left|\alpha_{j}\right|\left|\alpha_{k}\right|: \alpha_{j} \in J^{i}, \alpha_{k} \in J, \quad \alpha_{j}, \alpha_{k}\right. \text { approach each other } \\
\left.\quad \text { and } \alpha_{j}, \alpha_{k} \neq x_{1}^{l}(t), x_{2}^{l}(t)\right\} \quad i=1,2,3, & \\
M & =\max \left\{1+4 C_{1}\left|r^{0}\right|, 1+4 C_{1}\left|s^{0}\right|\right\}
\end{aligned}
$$

(here $\left|r^{0}\right|$ and $\left|s^{0}\right|$ are the strengths of $x_{1}(t)$ and $x_{2}(t)$, respectively, and $C_{1}$ is a constant appearing in Lemma 2.3).

In $L\left(J^{i}\right)$ and $Q\left(J^{i}\right), i=1,3, \alpha \in J^{i}$ seems to imply $\alpha \neq x_{j}^{l}(t), j=1,2$. In fact, when $J=J_{\left(t_{1}+h\right)}, \alpha \in J^{1}$ (or $J^{3}$ ) implies $\alpha=x_{1}^{l}(t)$ (or $\alpha=x_{2}^{l}(t)$ ). We stipulate that $F\left(J^{2}\right)=0$ when there is no wave crossing $J^{2}$.

(II) When $J$ is on $t \geq t_{1}$, we define on $J$ :

$$
\begin{aligned}
F_{2}^{(s)}(J) & =\sum_{i=1}^{3} F\left(J^{i}\right) \\
F\left(J^{i}\right) & =M_{i} L\left(J^{i}\right)+Q\left(J^{i}\right) \quad i=1,3 \\
F\left(J^{2}\right) & =L\left(J^{2}\right)+Q\left(J^{2}\right),
\end{aligned}
$$

where $J^{1}$ (or $J^{3}$ ) is the part of $J$ lying to the left (or right) of $x_{1}^{l}(t)$ (or $x_{2}^{l}(t)$ ), $J^{2}$ is the part of $J$ between $x_{1}^{l}(t)$ and $x_{2}^{l}(t), M_{1}=1+4 C_{1}\left|B_{1}^{0}\right|, M_{2}=1+4 C_{1}\left|B_{2}^{0}\right|$, $\left|B_{1}^{0}\right|=2\left(1+2 C_{1}\left|s^{0}\right|\right)\left|r^{0},\right| B_{2}^{0}\left|=2\left(1+2 C_{1}\left|r^{0}\right|\right)\right| s^{0} \mid$, and the rest of the definition is as in $(\mathrm{I})$.

If $x_{2}^{l}(t)$ does not interact with $x_{1}^{l}(t)$ forever, then there is no need to define $F_{2}^{(s)}(J)$.

We now sketch the proof of the conclusion that the total variation of the approximate perturbed solutions $\left(\bar{u}^{l}(\cdot, t), \bar{v}^{l}(\cdot, t)\right)$ is uniformly bounded for any $t \geq 0$. The details are omitted.

(I) Using the techniques similar to the ones for Theorem 3.1, for any $I$-curve $J$ on $0 \leq t \leq t_{1}+h$, we can inductively prove

$$
F_{1}^{(s)}(J)<\eta \quad(\eta=4 M \varepsilon)
$$

and the total sum of the strengths of all waves crossing $J$ is

$$
\begin{aligned}
L_{s}(J) & \leq\left|r^{0}\right|+\left|s^{0}\right|+F\left(J^{1}\right)+2 F\left(J^{2}\right)+F\left(J^{3}\right)+F_{1}^{(s)}(J) \\
& <\left|r^{0}\right|+\left|s^{0}\right|+3 \eta .
\end{aligned}
$$


(II) There is no difficulty in proving

$$
F_{2}^{(s)}\left(J_{\left(t_{1}+h\right)}\right)<\max \left\{M_{1}, M_{2}\right\} \eta
$$

(III) Let $t=t_{1}$ be the initial line and $J_{\left(t_{1}+h\right)}$ be the initial $I$-curve. Similar to Theorem 3.1, we have that the total sum of the strengths of all waves crossing $J$ is uniformly bounded for any $I$-curve $J$ on $t \geq t_{1}$.

Now, we shall discuss the case in which the unperturbed initial data $\left(u_{0}(x), v_{0}(x)\right)$ will generate a 2-shock wave and a 2-centered rarefaction wave lying to its right. For the case in which the unperturbed initial data will generate a 2-centered rarefaction wave and a 2 -shock wave lying to its right, we can study it similarly.

Theorem 3.4. Suppose that the unperturbed initial data $\left(u_{0}(x), v_{0}(x)\right)$ consist of three pieces of constant functions generating a 2-shock wave $x_{2}(t)$ and a 2-centered rarefaction wave, where $x_{2}(t)$ lies to the left of the 2-centered rarefaction wave and the strengths of the 2-shock wave $x_{2}(t)$ and 2-centered rarefaction wave are $\left|s^{0}\right|$ and $\left|g^{0}\right|$, respectively. If we give a small perturbation to $\left(u_{0}(x), v_{0}(x)\right)$, then there exists a perturbed solution of the corresponding unperturbed problem (E) and (I).

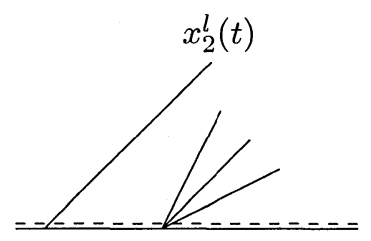

FiguRe 5

Proof. Let $x_{2}^{l}(t)$ denote the perturbed approximate shock wave of $x_{2}(t)$ (Figure 5). The perturbed 2-rarefaction wave which originated from $t=0^{+}$will be scattered in the time evolution. Because of the perturbation, some small 2-rarefaction waves will appear at $t=0^{+}$. The interaction of 2-rarefaction waves and $x_{2}^{l}(t)$ from the left or right will produce some new approximate 1 -shock waves. We denote by $\mathbf{S}$ the set of $x_{2}^{l}(t)$, these new approximate 1-shock waves and those approximate 1-shock waves produced by interactions among these new approximate 1-shock waves. Let $t=t_{i}$, $i=1,2, \ldots, N$ ( $N$ may be an arbitrary positive integer $)$ be the time at which 2 rarefaction waves orderly interact with $x_{2}^{l}(t)$ from the left or right. Let $\left|r_{i}^{0}\right|$ be the strength of the 1-shock wave produced by the interaction of the 2-rarefaction wave and $x_{2}^{l}(t)$ from the left or right at time $t=t_{i}, i=1,2, \ldots, N$, whose corresponding approximate 1 -shock wave is denoted by $x_{1}^{l_{i}}(t), i=1,2, \ldots, N$.

We now inductively design functional sequences $\left\{F_{i}^{(s)}\right\}_{i=1}^{N+1}$ and $\left\{F_{i}^{(L)}\right\}_{i=1}^{N+1}$ such that for any $I$-curve $J$ on $t_{i-1} \leq t \leq t_{i}$ where $1 \leq i \leq N+1, t_{0}=0$, the functional $F_{i}^{(s)}(J)$ dominates the total sum of the strengths of all shock waves crossing $J$ except those belonging to $\mathbf{S}$ and is uniformly small for $i$ and $J$, and $F_{i}^{(L)}(J)$ dominates 
the total sum of the strengths of the shock waves crossing $J$ belonging to $\mathbf{S}$ and is uniformly bounded for $i$ and $J$.

(I) When any $I$-curve $J$ is on $0 \leq t \leq t_{1}+h$ ( $h$ is time mesh), we define on $J$ :

$$
\begin{aligned}
F_{1}^{(s)}(J) & =F_{0}\left(J^{1}\right)+F_{0}\left(J^{N+2}\right), \\
F_{0}\left(J^{i}\right) & =L_{0}\left(J^{i}\right)+Q_{0}\left(J^{i}\right), \quad i=1, N+2, \\
L_{0}\left(J^{i}\right) & =\sum\left\{|\alpha|: \alpha \in J^{i}, \alpha \notin \mathbf{S}, \alpha \text { is not a 2-rarefaction wave }\right\}, \quad i=1, N+2, \\
Q_{0}\left(J^{i}\right) & =\sum\left\{\left|\alpha_{j}\right|\left|\alpha_{k}\right|: \alpha_{j} \in J^{i}, \alpha_{k} \in J, \alpha_{j}, \alpha_{k} \notin \mathbf{S},\right. \\
\alpha_{j}, \alpha_{k} \text { are not 2-rarefaction waves } & \quad \text { and approach each other }\}, \quad i=1, N+2, \\
F_{1}^{(L)}(J) & =L_{s}^{(L)}(J)+F_{1}^{(s)}(J)+L_{2 s}^{(s)}(J), \\
L_{s}^{(L)}(J) & =\sum\{|\alpha|: \alpha \in J \bigcap \mathbf{S}, \\
L_{2 s}^{(s)}(J) & =\sum\left\{|\alpha|: \alpha \in J, \alpha \text { is a 2-shock wave, and } \alpha \neq x_{2}^{l}(t)\right\} \\
M & =2+4 C_{1}\left|s^{0}\right|,
\end{aligned}
$$

where $J^{1}$ (or $J^{N+2}$ ) is the part of $J$ lying to the left (or right) of $x_{2}^{l}(t)$, and $C_{1}$ is a constant appearing in Lemma 2.3,

(II) When $J$ is on $t_{n} \leq t \leq t_{n}+h, n \leq N$ is arbitrary. It does no harm to suppose that there are no interactions among the waves belonging to $\mathbf{S}$ below $J$ and that each of these waves is not eliminated. Then the approximate shock waves $x_{1}^{l_{1}}(t), \ldots, x_{1}^{l_{n}}(t)$ belonging to $\mathbf{S}$ are all crossing $J$. Otherwise, our problem will be simpler. We define functionals on $J$ :

$$
\begin{aligned}
F_{n+1}^{(s)}(J) & =\sum_{i=1}^{n+1} F_{0}\left(J^{i}\right)+F_{0}\left(J^{N+2}\right), \\
F_{0}\left(J^{i}\right) & =M_{i} L_{0}\left(J^{i}\right)+Q_{0}\left(J^{i}\right), \quad i=1,2, \ldots, n+1,
\end{aligned}
$$

where $J^{1}$ is the part of $J$ lying to the left of $x_{1}^{l_{1}}(t), J^{i}, i=2,3, \ldots, n$, is the part of $J$ between $x_{1}^{l_{i-1}}(t)$ and $x_{1}^{l_{i}}(t), J^{n+1}$ is the part of $J$ between $x_{1}^{l_{n}}(t)$ and $x_{2}^{l}(t)$, and $J^{N+2}$ is the part of $J$ lying to the right of $x_{2}^{l}(t)$. The definition of $F_{0}\left(J^{N+2}\right)$ is as in (I).

$$
\begin{aligned}
L_{0}\left(J^{i}\right)= & \sum\left\{|\alpha|: \alpha \in J^{i}, \alpha \notin \mathbf{S}, \text { and } \alpha \text { is not a 2-rarefaction wave }\right\} \\
\qquad \begin{array}{l}
Q_{0}\left(J^{i}\right)= \\
\end{array} & \sum\left\{\left|\alpha_{j}\right|\left|\alpha_{k}\right|: \alpha_{j} \in J^{i}, \alpha_{k} \in J, \alpha_{j}, \alpha_{k} \notin \mathbf{S},\right. \\
& \left.\alpha_{j}, \alpha_{k} \text { are not 2-rarefaction waves and approach each other }\right\} \\
& i=1, \ldots, n+1, \\
M_{i}= & \prod_{j=i}^{n}\left(1+6 C_{1}\left|r_{j}^{0}\right|\right) \quad i=1,2, \ldots, n, \quad M_{n+1}=1, \\
F_{n+1}^{(L)}(J)= & L_{s}^{(L)}(J)+F_{n+1}^{(s)}(J)+L_{2 s}^{(s)}(J) .
\end{aligned}
$$

The definitions of $L_{s}^{(L)}(J)$ and $L_{2 s}^{(s)}(J)$ are as in (I). 
We now define another functional $F^{+}(J)$ to dominate the total sum of the strengths of those waves which interact with $x_{2}^{l}(t)$ from the right below any $I$-curve $J$ on the upper half plane. Let

$$
\begin{aligned}
F^{+}(J) & =L\left(J^{N+2}\right)+Q\left(J^{N+2}\right), \\
L\left(J^{N+2}\right) & =\sum\left\{|\alpha|: \alpha \in J^{N+2}\right\}, \\
Q\left(J^{N+2}\right) & =\sum\left\{\left|\alpha_{j}\right|\left|\alpha_{k}\right|: \alpha_{j}, \alpha_{k} \in J^{N+2}, \alpha_{j}, \alpha_{k} \text { approach each other }\right\}
\end{aligned}
$$

where $J^{N+2}$ is the part of $J$ lying to the right of $x_{2}^{l}(t)$, whose definition is consistent with the above.

Since a large 2-rarefaction wave issued from the initial line $t=0^{+}$and newly generated 2-rarefaction waves are not small, in order to guarantee that $F_{i}^{(s)}(J)$ is decreasing on $t_{i-1} \leq t \leq t_{i}$ and uniformly small for $i$ and $J$, we did not consider the strengths of 2-rarefaction waves in the definition of $F_{i}^{(s)}(J), 1 \leq i \leq N+1$. When a 2-shock wave not belonging to $\mathbf{S}$ interacts with $x_{2}^{l}(t)$ from the left, $L_{s}^{(L)}(J)+$ $F_{i}^{(s)}(J)$ is not decreasing; thus we add a term $L_{2 s}^{(s)}(J)$ in $F_{i}^{(L)}(J)$ to ensure that $F_{i}^{(L)}(J)$ is decreasing on $t_{i-1} \leq t \leq t_{i}, i=1,2, \ldots, N+1$. Here $F_{i}^{(s)}(J)$ and $F_{i}^{(L)}(J)$ decreasing on $t_{i-1} \leq t \leq t_{i}$ means that for any $I$-curve $J_{I}$ on $t_{i-1} \leq t \leq t_{i}$ and its immediate successor $J_{I I}$ on $t_{i-1} \leq t \leq t_{i}$, we have $F_{i}^{(s)}\left(J_{I I}\right) \leq F_{i}^{(s)}\left(J_{I}\right)$ and $F_{i}^{(L)}\left(J_{I I}\right) \leq F_{i}^{(L)}\left(J_{I}\right)$.

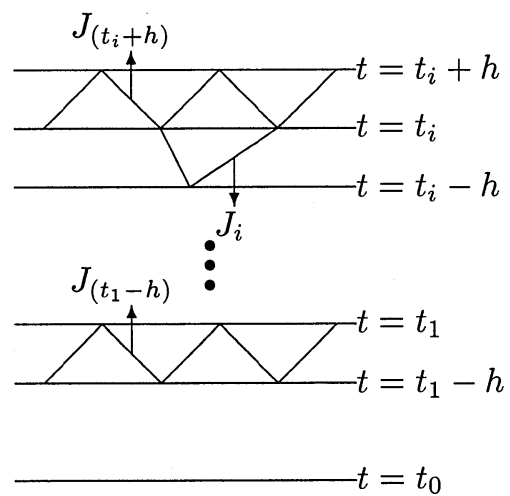

FiguRE 6

Let $J_{\left(t_{0}+h\right)}, J_{\left(t_{1}-h\right)}, J_{\left(t_{1}+h\right)}, \ldots, J_{\left(t_{N}-h\right)}, J_{\left(t_{N}+h\right)}$ denote the $I$-curves lying in $t_{0} \leq$ $t \leq t_{0}+h, t_{1}-h \leq t \leq t_{1}, t_{1} \leq t \leq t_{1}+h, \ldots, t_{N}-h \leq t \leq t_{N}, t_{N} \leq t \leq t_{N}+h$ respectively, where $t_{0}=0$ and $J_{\left(t_{0}+h\right)}=O$ (Figure 6). Let $J_{i}, i=1,2, \ldots, N$, denote the $I$-curve satisfying the condition that $J_{\left(t_{i}+h\right)}$ is an immediate successor to it, and in the diamond $\Delta$ bounded by it and $J_{\left(t_{i}+h\right)}$, a 2-rarefaction wave interacts with $x_{2}^{l}(t)$ from the left or right. At each time $t=t_{n}, 1 \leq n \leq N$, the interaction of $x_{2}^{l}(t)$ and the elementary wave lying to its left or right is either a 2-rarefaction wave overtaking $x_{2}^{l}(t)$ 
or $x_{2}^{l}(t)$ overtaking a 2-rarefaction wave, namely, a part of $\left|r_{i}^{0}\right|, i=1,2, \ldots, n$, coming from the 2-rarefaction waves which interact with $x_{2}^{l}(t)$ from the left, and another part coming from the 2-rarefaction waves which interact with $x_{2}^{l}(t)$ from the right. We relabel them. Those from the left are $\left|r_{l_{1}}^{0}\right|, \ldots,\left|r_{l_{p(n)}}^{0}\right|$; those from the right are $\left|r_{r_{1}}^{0}\right|, \ldots,\left|r_{r_{q(n)}}^{0}\right|$. The 2-rarefaction waves which interact with $x_{2}^{l}(t)$ from the right are denoted by $g_{r_{1}}, \ldots, g_{r_{q(n)}}$, respectively, where $p(n)+q(n)=n$.

In order to prove this theorem, we only need to prove for all $k \in Z^{+}$that:

(A) When any $I$-curve $J$ is on $t_{k-1} \leq t \leq t_{k}$,

$$
\begin{aligned}
F_{k}^{(s)}(J) & \leq F_{k}^{(s)}\left(J_{\left(t_{k-1}+h\right)}\right)<\eta, \\
F_{k}^{(L)}(J) & \leq F_{k}^{(L)}\left(J_{\left(t_{k-1}+h\right)}\right)<G, \\
F^{+}(J) & \leq F^{+}\left(J_{\left(t_{k-1}+h\right)}\right) \leq F^{+}(O)<W .
\end{aligned}
$$

(B) When $J$ is on $t_{k} \leq t \leq t_{k}+h$, i.e., $J=J_{\left(t_{k}+h\right)}$,

$$
\begin{aligned}
& F_{k+1}^{(s)}(J) \leq F_{1}^{(s)}(O) \prod_{j=1}^{k}\left(1+6 C_{1}\left|r_{j}^{0}\right|\right)<\eta \\
& F_{k+1}^{(L)}(J) \leq F_{1}^{(L)}(O)-\frac{C_{0}}{2} \prod_{j=1}^{p(k)}\left|r_{l_{j}}^{0}\right|+4 C_{1}\left|s^{0}\right| \sum_{j=1}^{q(k)}\left|g_{r_{j}}\right|<G, \\
& \sum_{j=1}^{q(k)}\left|r_{r_{j}}^{0}\right| \leq 2 C_{1}\left|s^{0}\right| \sum_{j=1}^{q(k)}\left|g_{r_{j}}\right|, \\
& \sum_{j=1}^{q(k)}\left|g_{r_{j}}\right| \leq F^{+}(O)-F^{+}(J) \leq F^{+}(O)<W
\end{aligned}
$$

where $W=\left(\left|g^{0}\right|+2 \varepsilon\right)^{2}+\left|g^{0}\right|+2 \varepsilon, G=\left(4 C_{1} W+1\right)\left|s^{0}\right|+10 M \varepsilon$,

$$
\eta=4 M \varepsilon \exp \left\{\frac{12 C_{1}}{C_{0}}\left(\left(1+4 C_{1} W+C_{0} C_{1} W\right)\left|s^{0}\right|+10 M \varepsilon\right)\right\}
$$

$C_{0}, C_{1}$ are constants appearing in Lemma 2.3 , and $\varepsilon$ is a sufficiently small constant appearing in (3.3).

We shall prove conclusions (A) and (B) by induction. We only give the main steps below. The details are omitted here.

(I) We prove (A) and (B) are valid for $k=1$.

(i) By considering all possible types of interactions of waves, it is not difficult to show that for any $I$-curve $J$ on $0 \leq t \leq t_{1}, F_{1}^{(s)}(J)$ and $F^{+}(J)$ are decreasing and the strength of $x_{2}^{l}(t)$ crossing $J$ satisfies

$$
\begin{aligned}
\left|x_{2}^{l}(t)\right| & \leq\left|s^{0}\right|+2 \varepsilon+\sum^{r}(|\beta|+|\gamma|)+\sum^{\ell}|\gamma| \\
& <\left|s^{0}\right|+2 \varepsilon+2 D\left(F_{1}^{(s)}(O)-F_{1}^{(s)}(J)\right) \\
& <2\left|s^{0}\right|
\end{aligned}
$$


where $\sum^{r}(|\beta|+|\gamma|)$ denotes the total sum of the strengths of 1-shock waves and 2-shock waves which interact with $x_{2}^{l}(t)$ from the right below $J$ and $\sum^{\ell}|\gamma|$ denotes the total sum of the strengths of 2-shock waves which interact with $x_{2}^{l}(t)$ from the left below $J$.

Therefore, we can prove $(\mathrm{A})$ is valid for $k=1$.

(ii) When the $I$-curve $J=J_{\left(t_{1}+h\right)}$, then at time $t=t_{1}$, the interaction is either $g+\gamma \rightarrow \beta^{\prime}+\gamma^{\prime}\left(\gamma=x_{2}^{l}(t)\right)$ or $\gamma+g \rightarrow \beta^{\prime}+\gamma^{\prime}\left(\gamma=x_{2}^{l}(t)\right)$ in the diamond $\Delta$ bounded by $J_{\left(t_{1}+h\right)}$ and $J_{1}$. If the interaction is $g+\gamma \rightarrow \beta^{\prime}+\gamma^{\prime}\left(\gamma=x_{2}^{l}(t)\right)$, then $\left|r_{l_{1}}^{0}\right|=\left|r_{1}^{0}\right|=\left|\beta^{\prime}\right|$, $\left|r_{r_{1}}^{0}\right|=0,\left|g_{r_{1}}\right|=0$, and $\mid x_{2}^{l}(t)$ crossing $J|<2| s^{0} \mid$. If the interaction is $\gamma+g \rightarrow \beta^{\prime}+\gamma^{\prime}$ $\left(\gamma=x_{2}^{l}(t)\right)$, then $\left|r_{l_{1}}^{0}\right|=0,\left|r_{r_{1}}^{0}\right|=\left|r_{1}^{0}\right|=\left|\beta^{\prime}\right|, g_{r_{1}}=g$, and $\mid x_{2}^{l}(t)$ crossing $J|<2| s^{0} \mid$. Thus we can prove the conclusion (B) is valid for $k=1$.

(II) Suppose that the conclusions (A) and (B) are valid for $k=n$. We need to prove that $(\mathrm{A})$ and $(\mathrm{B})$ are valid for $k=n+1$.

Let $t=t_{n}$ be the initial line and $J_{\left(t_{n}+h\right)}$ is the initial $I$-curve.

(i) When any $I$-curve $J$ is on $t_{n} \leq t \leq t_{n+1}$, by considering all possible types of interactions of waves, we have that $F_{n+1}^{(s)}(J), F_{n+1}^{(L)}(J)$, and $F^{+}(J)$ are decreasing and the strength of $x_{2}^{l}(t)$ crossing $J$ satisfies:

$$
\begin{aligned}
\left|x_{2}^{l}(t)\right| & \leq\left|s^{0}\right|+2 \varepsilon+\sum^{r}(|\beta|+|\gamma|)+\sum^{\ell}|\gamma| \\
& <\left|s^{0}\right|+2 \varepsilon+2 D\left(F_{1}^{(s)}(O)+6 C_{1} \eta \sum_{i=1}^{n}\left|r_{i}^{0}\right|-F_{n+1}^{(s)}(J)\right) \\
& <2\left|s^{0}\right| .
\end{aligned}
$$

Therefore, using the results that $(\mathrm{A})$ and $(\mathrm{B})$ are valid for $k=n$, we can prove (A) is valid for $k=n+1$.

(ii) When the $I$-curve $J=J_{\left(t_{n+1}+h\right)}$, then at time $t=t_{n+1}$, the interaction is either $g+\gamma \rightarrow \beta^{\prime}+\gamma^{\prime}\left(\gamma=x_{2}^{l}(t)\right)$ or $\gamma+g \rightarrow \beta^{\prime}+\gamma^{\prime}\left(\gamma=x_{2}^{l}(t)\right)$ in the diamond $\Delta$ bounded by $J_{\left(t_{n+1}+h\right)}$ and $J_{n+1}$.

If the interaction is $g+\gamma \rightarrow \beta^{\prime}+\gamma^{\prime}\left(\gamma=x_{2}^{l}(t)\right)$, then $\left|r_{l_{p(n+1)}}^{0}\right|=\left|r_{n+1}^{0}\right|=\left|\beta^{\prime}\right|$, $\left|r_{r_{q(n+1)}}^{0}\right|=0,\left|g_{r_{q(n+1)}}\right|=0$, and the strength of $x_{2}^{l}(t)$ crossing $J$ is less than $2\left|s^{0}\right|$.

If the interaction is $\gamma+g \rightarrow \beta^{\prime}+\gamma^{\prime}\left(\gamma=x_{2}^{l}(t)\right)$, then $\left|r_{l_{p(n+1)}}^{0}\right|=0,\left|r_{q(n+1)}^{0}\right|=$ $\left|r_{n+1}^{0}\right|=\left|\beta^{\prime}\right|, g_{r_{q(n+1)}}=g$, and the strength of $x_{2}^{l}(t)$ crossing $J$ is less than $2\left|s^{0}\right|$.

Consequently, using the results obtained in (i) and the result that (B) is valid for $k=n$, we also can prove (B) is valid for $k=n+1$.

\section{References}

1. F. Asakura, Asymptotic stability of solutions with a single strong shock wave for hyperbolic systems of conservation laws, Japan J. Indust. Appl. Math. 11 (1994), 225-244.

2. - Decay of solutions for the equations of isothermal gas dynamics, Japan J. Indust. Appl. Math. 10 (1993), 133-164.

3. ___ Asymptotic stability of solutions with strong discontinuities for the equations of isothermal gas dynamics, Japan J. Indust. Appl. Math. 11 (1994), 427-464.

4. I-L. Chern, Stability theorem and truncation error analysis for the Glimm scheme and for a front tracking method for flows with strong discontinuities, Comm. Pure Appl. Math. XLII (1989), 815-844.

5. S. S. Ding, T. Chang, C. H. Wang, L. Hsiao, and T. C. Li, A study of the global solutions for quasi-linear hyperbolic systems of conservation laws, Scientia Sinica XVI (1973), 317-335. 
6. J. Glimm, Solutions in the large for nonlinear hyperbolic systems of equations, Comm. Pure Appl. Math. 18 (1965), 95-105.

7. J. Johnson and J. Smoller, Global solutions of certain hyperbolic systems of quasi-linear equations, J. Math. Mech. 17 (1967), 561-576.

8. L. W. Lin and F. P. Xie, Perturbation of rarefaction waves, Acta Math. Sinica, New Series 8 (1992), 122-134.

9. T. P. Liu, Asymptotic behavior of solutions of general system of nonlinear hyperbolic conservation laws, Indiana. Math. J. 27 (1987), 211-253.

10. T. Nishida and J. Smoller, Solutions in the large for some nonlinear hyperbolic conservation laws, Comm. Pure Appl. Math. 26 (1973), 183-200.

11. J. Smoller and J. Johnson, Global solutions for an extended class of hyperbolic systems of conservation laws, Arch. Rat. Mech. Anal. 32 (1969), 169-189.

12. B. Temple, On supnorm bounds in Glimm's method, Math. University of Wisconsin Madsion.

13. C. H. Wang and C. Z. Li, Study of the global solutions for nonlinear conservation laws, J. Math. Anal. Appl. 85 (1982), 236-256.

14. T. Zhang and Y. F. Guo, A class of initial-value problems for systems of aerodynamic equations, Acta Math. Sinica, 15 (1965), 386-396; English translation in Chinese Math. 7 (1965), 90-101.

Department of Mathematics, University of Jinan, Guangzhou, 510632, China

Department of Mathematics, University of Macau, Macau 